\title{
Transform and statistical parameter-based image noise level prediction
}

\author{
K. Sivakumar \\ Asst. Prof., Department of Electronics and Communication Engineering \\ Rajalakshmi Institute of Technology, Chennai,
}

\begin{abstract}
Noise level estimation in an image is important and useful in many image processing algorithms such as image de-noising, image segmentation and image compression. Accurately estimating the noise level without the prior knowledge of the image is the major challenge of today's research. We present an improved patch based fast noise level estimation using DCT and standard deviation method for fast and reliable noise level estimation and the result is compared with the available state-of-art methods. Experimental result shows the proposed method provides greater accuracy, the stability and also the proposed method is an average of six times faster than that of the state- of - art methods for noise level estimation.
\end{abstract}

Keywords: Noise level estimation, DCT Basis, White Gaussian Noise

\section{INTRODUCTION}

Noise level is significant parameter in image processing algorithms that include image de-nosing [1], [2], [3], [4], [5] [6], image segmentation [7], [8], [9], Image compression[13], Super resolution [10], Optical flow[11],[12], Image quality assessment[14],[15]. Because of the local variance of the image, that may be due to the noise present with the image, or due the image texture, from the single image accurately estimating the noise level is a challenging one. Available algorithm for estimating the noise level is not suitable when there is a more percentage of noise with an image or very less percentage of noise with the image. The primary step in noise level estimation is to prepare the data set. Considering data set preparation as reference or the key point the existing algorithms can broadly be classified in to three categories. Noise level estimation based on transformation, based on filtering and based on small mask.

The transform-based methods, [16], [17], [18], image is being transformed in the frequency domain and then estimates the noise level. Donoho and Johnstone [16] method applies wavelet transform to isolate the noise and then estimate the noise level from the wavelet coefficient. This method cannot be used for high frequency image since it provides overestimated noise level.

In filter based approach, Russo. F [22], Tai, S.C., Yang[23], the image is first filtered then difference of the original and the filtered image is considered for noise variance estimation with assumption that the difference image is pure noise. But this is not true in all the reality and this method works well for the image with less texture and at the same time overestimates for the high frequency images.

Huang, X.et.al [24], Liu, X et.al [25], S. Pyatykh et.al, [26] and Daniel Zoran [29] has presented patch based noise level estimation method. Noise variance is estimated on the identified homogenous patches and major challenge with patch based method is to identifying the homogenous patches. Proposed method is based on [29] but it achieves better performance in terms of stability, accuracy and processing time, and it neglects complex calculation like solving minimization function and finding the kurtosis etc. as done in [29] and the experimental result is compared with state of art methods [25], [26] and [29] and along with the performance parameter additionally one more parameter, called estimation ratio, is also used to prove the strength of the proposed method and that also compared with [25],[26] and [29] and from the estimation noise ratio it is very clear that the proposed method outstandingly estimates the noise level in an image 
The rest of the paper is organized as follows. Section II, a brief discussion on methods related to the research, Section III, the detailed description of proposed method, Section IV and $\mathrm{V}$ respectively experimental result and conclusion.

\section{RELATED WORKS}

In the year 2009, Daniel Zoran et al [29] introduced a method to estimate noise variance which is based on the scale invariant property of the natural images. The assumption and method presented in [29] as follows

Let $x$ be the noise free image and is considered as the generalized Gaussian random variable and $\eta$ be the independent Gaussian random variable with zero mean and variance $\sigma_{\eta}^{2}$ and then the noisy image is represented by,

$y=x+\eta$

The fourth central moment, called kurtosis, is referred and is normalized by the variance squared and is given by

$$
k=\frac{\mu_{4}}{\sigma^{4}}
$$

Where $\mu$ is the mean and

$\sigma^{2}$ is the variance.

Actual noise level is estimated, by minimizing the function given in (3), by finding the kurtosis of the original uncorrupted image $\hat{k}_{x}$ and the variance of the noise $\hat{\sigma}_{n}^{2}$, given kurtosis and variance. The minimal function given in [29] is

$$
\hat{k}_{x}, \hat{\sigma}_{\eta}^{2}=\arg \min \sum_{i=2}^{N^{2}}\left|\frac{k_{x}-3}{\left(1+\frac{\sigma_{\eta}^{2}}{\hat{\sigma}_{y i}^{2}-\sigma_{\eta}^{2}}\right)}+3-\sigma_{y i}^{2}\right|
$$

In the year 2012,S. Pyatykh et al [26] presented Principal Component Analysis (PCA) based method to estimate noise level. Noise variance is calculated from the covariance matrix of image block with smallest Eigen value. S. Pyatykh et al method shows very good tradeoff between speed and accuracy further it does not assume any homogeneous areas. The algorithm proposed in [26] as follows.

Assume the noisy image as $y$ and corrupted by noise $\eta$ with variance $\sigma_{\eta}^{2}$.

Assume the initial estimated noise variance as $\sigma_{\text {est }}^{2}$ and let upper bound of $y$ and it variance be $\sigma_{u b}^{2}$.

For each component $i$ with the inputs $\left(y, \sigma_{e s t}^{2}, \sigma_{u b}^{2}\right)$ calculate $\sigma_{\text {next }}^{2}$.

Stop the process if $\sigma_{\text {est }}^{2}$ is equal to $\sigma_{\text {next }}^{2}$, otherwise continue the process until $\sigma_{\text {est }}^{2}=\sigma_{\text {next }}^{2}$.

With this algorithm and algorithm named "GetNextEstimate", S. Pyatykh et al [26] has achieved good accuracy and stability performance in noise level estimation and is considered as one the state-of- art method by many of the researchers in the field.

In the year 2013, Liu, X et.al [25] proposed a texture strength metric of the gradient image and Noise level function to estimate noise parameter from single image with signal dependent noise. Without the priori information of the noise type the method in [25] will estimate the noise level.

\section{PROPOSED METHOD}

Since the existing methods suffer when the noise percentage is very high or noise percentage is considerablyvery low and also takes more processing time. Therefore, we introduce a method that estimates the noise level with more accurate and less processing time 
than that of Liu, X et.al [25], S. Pyatykh et al [26] and Daniel Zoran et al [29] methods. The proposed method is based the method presented in [29] but considerable amount of complexity and processing time is reduced and at the same time it ensures the accuracy, stability and produces comparable result with less processing time.

\subsection{Proposed method to estimate noise level}

Let $I_{x}$ be the noise free image and is considered as the generalized Gaussian random variable and noise added with the image is a white Gaussian noise with zero mean and variance $\sigma_{\eta}^{2}$ and then the noisy image is represented by,

$I_{y}=I_{x}+\eta$

Steps followed in proposed methods as follows,

Step1: start the noise level estimation with the assumption that the initial noise level is $\hat{\sigma}_{\eta}^{0}$

Step 2: Construct the DCT basis [32].

DCT can be defined, in general, for a 2D function with coordinates (x, y), as

(5)

$$
C(u, v)=\alpha(u) \alpha(v) \sum_{x=0}^{N-1} \sum_{y=0}^{N-1} f(x, y) \cos \left[\frac{\pi(2 x+1) u}{2 N}\right] \cos \left[\frac{\pi(2 y+1) v}{2 N}\right]
$$

\section{Where}

For $\mathrm{u}, \mathrm{v}=0,1,2 \ldots \mathrm{N}-1$ and $\alpha(\mathrm{u}) \alpha(\mathrm{v})$ are defined as

$$
\alpha(u)=\left\{\begin{array}{lll}
\sqrt{\frac{1}{N}}, & \text { for } & u=0 \\
\sqrt{\frac{2}{N}}, & \text { for } & u \neq 0
\end{array}\right.
$$

Step 3: Convolve the $2 \mathrm{D}$ input functions with DCT basis matrix developed using the equation (5) Shape parameter "Valid" is used while Convolving with DCT matric.

Step 4: Find the standard deviation of the result of convolution.

Step 5: Estimated noise level is equal to the minimum value of standard deviation obtained in step 4

Step 6: end the process.

The strength of proposed method in comparing with [29], the proposed method does not require the calculation of kurtosis and solving minimization function, results reduced amount of computational complexity so that it produces remarkable result in terms of improved accuracy and stability as well as less processing time. From experimental result the proposed method yields comparably good result to that of [25], [26]. To verify the stability and accuracy of proposed method we tried with different patch sizes wise 3x3, 5x5,7x7, 9x9 and $11 \times 11$. As mentioned in [29], we have taken $8 \times 8$ is the optimal patch size and the same is considered for comparison with existing methods.

\section{EXPERIMENTAL RESULT AND DISCUSSION}

To evaluate the performance of the proposed method 100 images of size $512 \times 512$ were used and were taken from https://www2.eecs.berkeley.edu/Research/Projects/CS/vision/_ sds/BSDS300/html/dataset/images/color/test-026-050.html with ref [30]. To have an idea about the noise free image and images with different percentage of noise, we have randomly chosen around ten images from the data base and have given for visual comparison and we named, on the top right corner, group of noise free image as "a”, image group with $5 \%$ noise as "b", image group with $25 \%$ noise as "c" and image group with $50 \%$ noise as "d" 
Table. 1 The noise level estimation accuracy performance comparison $\left(\mu_{\epsilon}\right)$

\begin{tabular}{|c|c|c|c|c|c|c|c|c|c|c|}
\hline \multirow{2}{*}{ Methods } & \multicolumn{10}{|c|}{ Noise Level $(\sigma)$} \\
\hline & 5 & 10 & 15 & 20 & 25 & 30 & 35 & 40 & 45 & 50 \\
\hline Proposed & 0.0309 & 0.0657 & 0.0793 & 0.0744 & 0.1221 & 0.1931 & 0.1014 & 0.1463 & 0.1512 & 0.3457 \\
\hline Ref[25] & 0.4300 & 0.5615 & 0.7310 & 0.8986 & 1.0073 & 0.8154 & 0.9231 & 0.8975 & 0.8975 & 1.1565 \\
\hline Ref[26] & 0.0408 & 0.0935 & 0.1965 & 0.2222 & 0.3157 & 0.3852 & 0.4792 & 0.5057 & 0.5321 & 0.6557 \\
\hline Ref[29] & 0.0969 & 0.1415 & 0.2102 & 0.2473 & 0.2473 & 0.4891 & 0.5251 & 0.6770 & 0.6855 & 0.7615 \\
\hline
\end{tabular}

Table. 2 Noise level estimation stability performance comparison $\left(\sigma_{\epsilon}^{2}\right)$

\begin{tabular}{|c|c|c|c|c|c|c|c|c|c|c|}
\hline \multirow{2}{*}{ Methods } & \multicolumn{10}{|c|}{ Noise Level $(\sigma)$} \\
\hline & 5 & 10 & 15 & 20 & 25 & 30 & 35 & 40 & 45 & 50 \\
\hline Proposed & 0.0002 & 0.0009 & 0.0013 & 0.0053 & 0.0092 & 0.0134 & 0.0057 & 0.0098 & 0.0189 & 0.0543 \\
\hline Ref[25] & 0.2349 & 0.3914 & 0.5670 & 0.8092 & 1.1460 & 0.5725 & 1.0465 & 0.6552 & 2.8440 & 2.995 \\
\hline $\operatorname{Ref[26]}$ & 0.0012 & 0.0292 & 0.0292 & 0.0160 & 0.0326 & 0.0677 & 0.0847 & 0.1014 & 0.0850 & 0.1310 \\
\hline $\operatorname{Ref[29]}$ & 0.0137 & 0.0233 & 0.0428 & 0.0580 & 0.1518 & 0.2230 & 0.2648 & 0.4406 & 0.4721 & 0.5618 \\
\hline
\end{tabular}

Table. 3 Estimation Ratio between actual noise levels to the estimated noise level

\begin{tabular}{|c|c|c|c|c|c|c|c|c|c|c|}
\hline \multirow{2}{*}{ Methods } & \multicolumn{10}{|c|}{ Noise Level $(\sigma)$} \\
\hline & 5 & 10 & 15 & 20 & 25 & 30 & 35 & 40 & 45 & 50 \\
\hline Proposed & 1.0013 & 1.0031 & 1.0007 & 1.001 & 0.9994 & 1.0004 & 0.9997 & 0.9989 & 1.0003 & 1.0002 \\
\hline Ref[25] & 0.9806 & 0.9803 & 0.9824 & 0.9836 & 0.9812 & 0.9819 & 0.982 & 0.9813 & 0.9803 & 0.9808 \\
\hline Ref[26] & 1.0285 & 1.003 & 0.9945 & 0.9924 & 0.9842 & 0.9872 & 0.9859 & 0.9834 & 0.9833 & 0.9831 \\
\hline Ref[29] & 0.9487 & 0.9478 & 0.9541 & 0.9559 & 0.9629 & 0.9754 & 0.9748 & 0.9769 & 0.9755 & 0.9802 \\
\hline
\end{tabular}

Table. 4 Average processing time of each noise estimation method for a 512x512 image

\begin{tabular}{|c|c|c|c|c|c|c|c|c|c|c|}
\hline \multirow{2}{*}{ Methods } & \multicolumn{10}{|c|}{ Noise Level $(\sigma)$} \\
\hline & 5 & 10 & 15 & 20 & 25 & 30 & 35 & 40 & 45 & 50 \\
\hline Proposed & 8.23 & 10.01 & 7.75 & 9.75 & 7.97 & 11.14 & 11.18 & 8.00 & 9.75 & 9.18 \\
\hline Ref[25] & 26.22 & 27.42 & 25.69 & 28.89 & 30.16 & 29.03 & 29.47 & 30.58 & 29.1 & 29.31 \\
\hline Ref[26] & 80.01 & 81.14 & 80.11 & 80.81 & 82.89 & 80.13 & 79.81 & 80.45 & 85.95 & 82.63 \\
\hline Ref[29] & 34.9 & 66.62 & 64.8 & 65.4 & 64.41 & 70.9 & 70.17 & 71.46 & 64.262 & 63.83 \\
\hline
\end{tabular}

Table 5 Overall performance estimation and comparison by MSE $=\sqrt{\mu_{\epsilon}^{2}+\sigma_{\epsilon}^{2}}$

\begin{tabular}{|c|c|c|c|c|c|c|c|c|c|c|}
\hline \multirow{2}{*}{ Methods } & \multicolumn{10}{|c|}{ Noise Level $(\sigma)$} \\
\hline & 5 & 10 & 15 & 20 & 25 & 30 & 35 & 40 & 45 & 50 \\
\hline Proposed & 0.0339 & 0.0722 & 0.0871 & 0.1040 & 0.1552 & 0.2251 & 0.1264 & 0.1766 & 0.2043 & 0.4169 \\
\hline Ref[25] & 0.1223 & 0.2047 & 0.2718 & 0.3624 & 0.4867 & 0.5782 & 0.6526 & 0.7609 & 0.9063 & 0.9733 \\
\hline Ref[26] & 0.1514 & 0.1212 & 0.1303 & 0.2086 & 0.4366 & 0.4009 & 0.5027 & 0.7013 & 0.7945 & 0.8843 \\
\hline Ref[29] & 0.5192 & 0.6712 & 0.8110 & 1.0562 & 1.1636 & 0.9441 & 1.1086 & 1.1904 & 1.5402 & 1.5287 \\
\hline
\end{tabular}

\subsection{Performance analysis}

Performance analysis Accuracy, Reliability, Estimation ratio and MSE are the four evaluation metrics considered to evaluate the performance of the noise estimation methods. The accuracy of the noise estimation method is identified by average estimation error and is given by the equation (6) 
$\mu_{\epsilon}=\frac{\sum_{i=1}^{M} E(i)}{M}$

where is the estimation error for the simulation and is defined as the difference between the actual and estimated noise level and $\mathrm{M}$ is the number of simulations. Average estimation error is tabulated, Table 2, for proposed as well as the methods considered for comparison, smaller the estimation error higher the accuracy of estimation of noise level and smaller estimation error is given in bold face. It is obvious from Figure 2 the estimation error plotted between actual noise level and average error and is minimum for proposed method than to that of the other state -of-art methods and an average estimation error achieved is 0.13 .

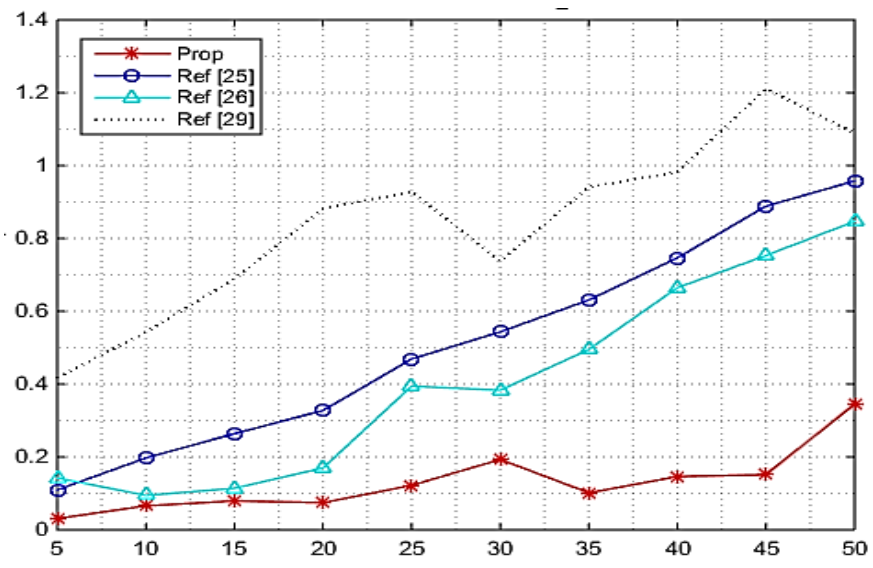

Figure. 1 Noise level versus average estimation error for different noise level

Average error variance is the metric used to ensure the stability of the noise level estimation method and is given by the equation (7), stability is ensured by smaller value of noise error variance and is comparably minimum for the proposed method than that of the existing methods Table 3 gives the error variance of the proposed and the existing methods, minimum error variance is given in bold face and from the figure, Figure 3, it is evident that proposed method archives minimum error variance.

$\sigma_{\epsilon}^{2}=\frac{\sum_{i=1}^{M}\left(E(i)-\mu_{\epsilon}\right)^{2}}{M}$

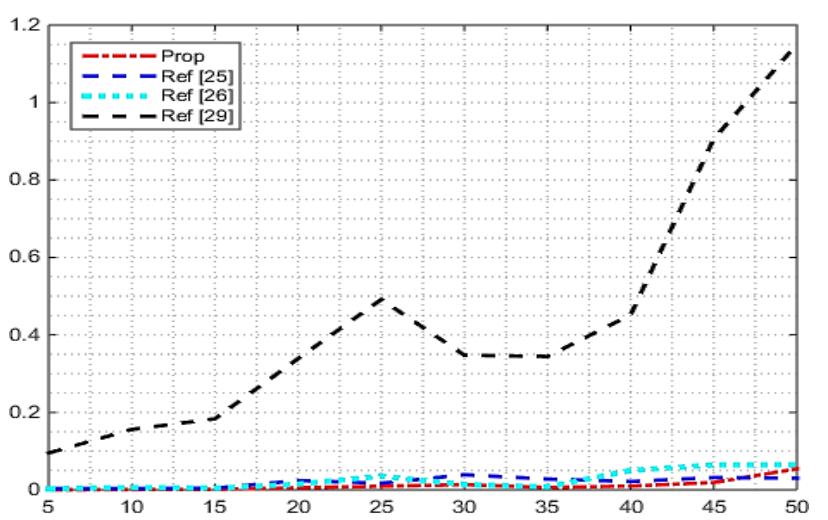

Figure. 2 Added noise level versus average error variance for different noise levels of each methods

Estimation noise ratio is the ratio of estimated noise level and the actual added noise level to the image, given in equation (8) and is one for perfect noise level estimation method, from the tabulated value, Table 3 and Figure 4, estimation error ratio is close to the ideal value for the proposed method than the existing methods for noise level estimation. 


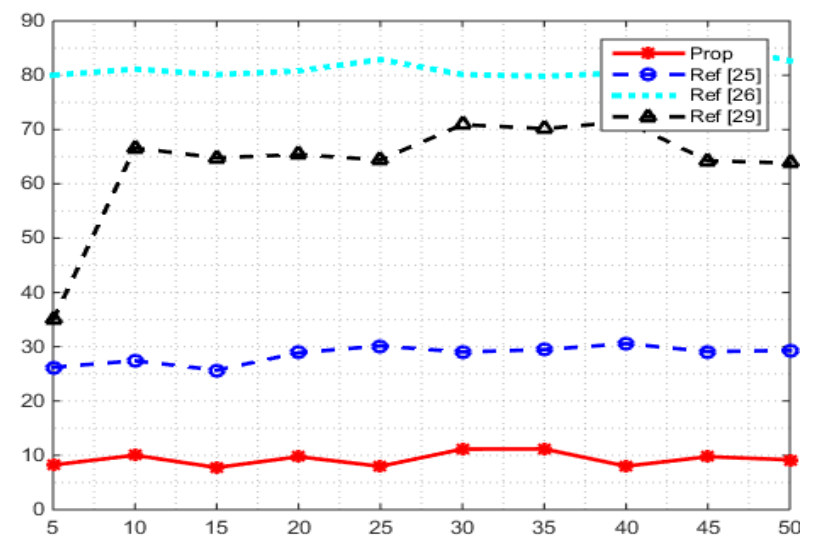

Figure 3 Noise level versus estimation ratio for proposed and existing methods

$$
\text { Estimation ratio }=\frac{\sigma_{\text {estimated }}}{\sigma_{\text {added }}}
$$

For all noise level from $5 \%$ to $50 \%$, the estimation ratio is calculated and is almost constant that is very closer to the ideal value, this ensures the proposed method is more robust irrespective of the noise level present in the image. To verify the overall performance of the noise level estimation method the metric called MSE is used and is given in equation (9) and MSE is tabulated for all the noise level estimation method and from the table, Table 5, proposed method archives smaller MSE value for all noise level, 5\% to 50\%, and smaller MSE indicates more accuracy and reliability of the estimation methods.

$$
\mathrm{MSE}=\sqrt{\mu_{\epsilon}^{2}+\sigma_{\epsilon}^{2}}
$$

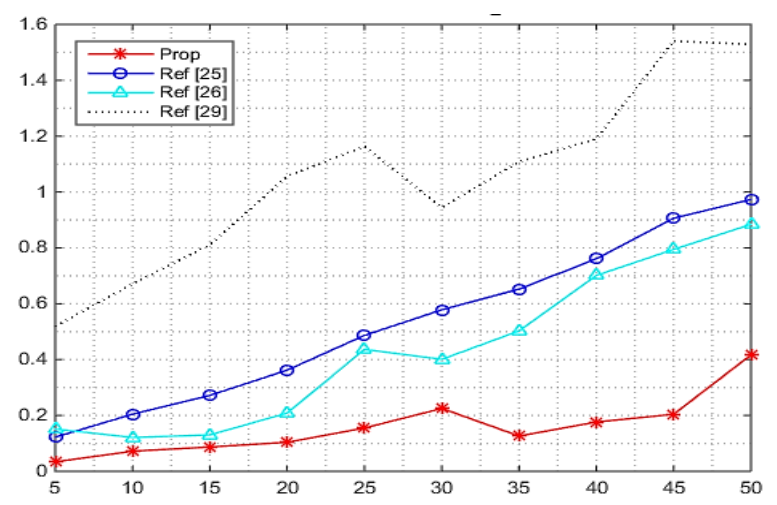

Figure 4 MSE for different noise level of proposed and other existing methods

The success of any image processing algorithms lies in less processing time and there is always a trade of between processing time and the best result. Average processing time of each noise estimation method to each noise level has been tabulated, Table 4, and the minimum processing time is in bold face. The average processing time of the proposed method is 9.30052s. The proposed method is 3.0736 times, 8.751s times and 6.846s times respectively faster than that of [25], [26] and [29]. It is the major advantages of the proposed method than that of the other existing methods. Figure 4 shows the proposed method yields best result with less processing time. Estimated noise level for each actual noise 


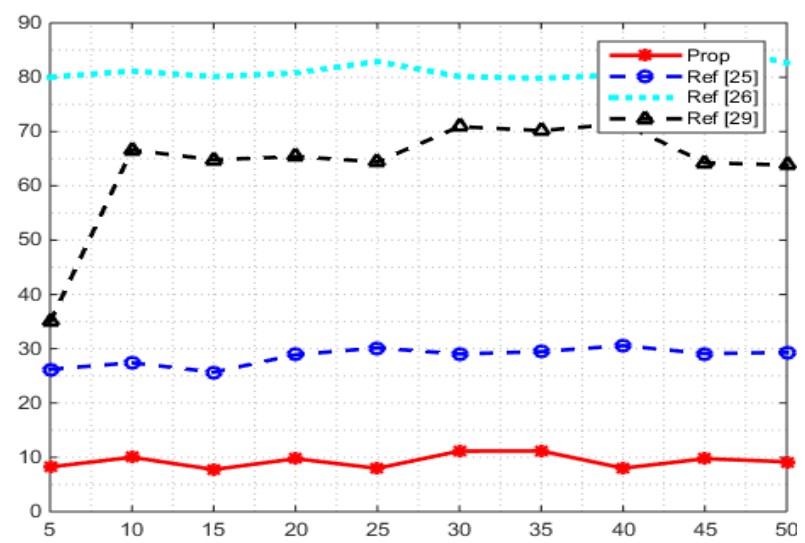

Figure 5 A comparative chart of average processing time for each noise level
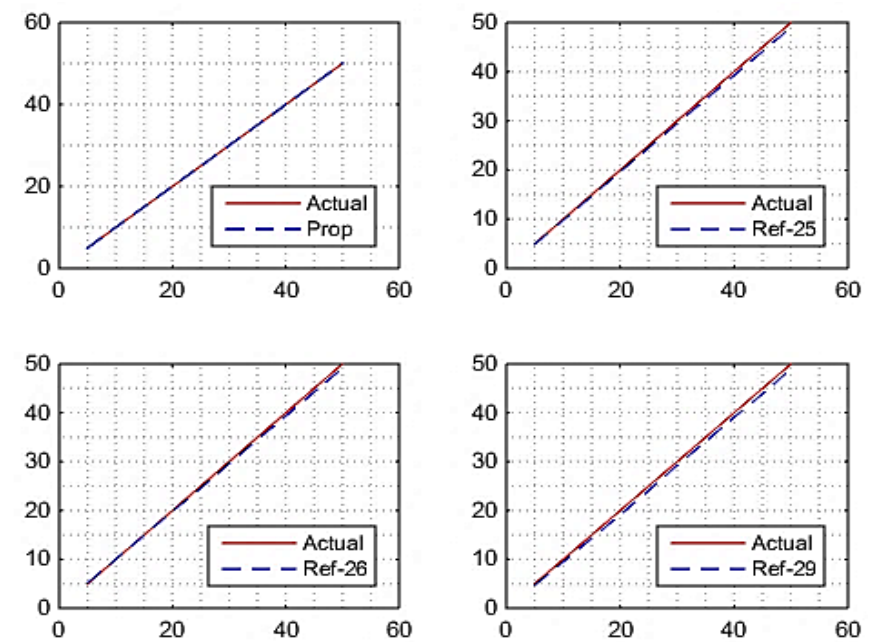

Figure 6. Added noise \% to the image and the estimated noise \% for each of the noise estimation methods, (a) Proposed method (Top - left), (b) Ref [25] (top- right), (b) Ref [26] (bottom- right), (b) Ref [29] (bottom- right)

added to the image is calculated and it plotted, Figure 5, shows the efficiency of the proposed method and for the proposed method the estimated noise level is almost close to the actual noise level ,5\% to 50\%, but for the other existing methods [25], [26] estimated noise is close to the actual noise level till $20 \%$ and there is deviation beyond $20 \%$ of the added noise and the variation is clear from the Figure 4 and from Table 2, Table 3.

Figure 4 clearly indicates the robustness of the proposed method in estimating the noise level present in the image. For the simulation of our proposed method as well as the ref [25], [26] and [29] we used the HP Pavilion 15 Notebook PC with Windows 10 home 64-bit operating system having the following specification intel(R) Core(TM) i5-4210 CPU @1.70GHz and 12 GB RAM. Simulation tool used: MATLAB, Version: 8.4.0.150421 (R2014b)

\section{CONCLUSION}

Since there is always a trade of between the expected result and the processing time but the in the case of noise level estimation the proposed method achieves best result that is smaller average error, average error variance and MSE with less processing time. This means that the proposed methods balances both run time as well as expected result than that of the other existing noise estimation methods. 
With respect to processing time the proposed method is 3.0736 times, 9.30052 times and 6.846 times respectively faster than that of [25], [26] and [29]. The proposed method estimates noise level with negligible percentage of estimation error for all added noise level over the range $5 \%$ to $50 \%$. Therefore the proposed method assures the accuracy, stability and balanced processing time. With this method for accurately estimating noise level, removing noise from the image can be improved.

\section{REFERENCES}

[1] A. Buades, B. Coll, and M. Morel. A review of image denoising algorithms, with a new one. MultiscaleModeling\& Simulation, 4(2):490-530, 2005.

[2] K. Dabov, A. Foi, V. Katkovnik, and K. Egiazarian. Image denoising with blockmatching and 3rd filtering. In Electronic Imaging 2006, pages 606414-606414. International Society for Optics and Photonics, 2006.

[3] K. Dabov, A. Foi, V. Katkovnik, and K. Egiazarian. Image denoising by sparse 3-d transform domain collaborative filtering. Image Processing, IEEE Transactions on, 16(8):2080-2095, 2007.

[4] M. Elad and M. Aharon. Image denoising via sparse and redundant representations over learned dictionaries. Image Processing, IEEE Transactions on, 15(12):37363745, 2006.

[5] M. Lebrun, A. Buades, and M. Morel. A nonlocal bayesianimage denoising algorithm. SIAM Journal on ImagingSciences, 6(3):1665-1688, 2013.

[6] Gilboa, G., Sochen, N., Zeevi, Y.Y., 2006. Estimation of optimal PDE-based denoising in the SNR sense. IEEE Trans. Image Process. 15, 2269-2280.

[7] P. Arbelaez, M. Maire, C. Fowlkes, and J. Malik. Contour detection and hierarchical

image segmentation. IEEE Trans. Pattern Anal. Mach. Intell., 33(5):898-916, May 2011.

[8] G. Chen, P.-A. Heng, and L. Xu. Projection-embedded by learning algorithm for Gaussian mixture-based clustering. In Applied Informatics, volume 1, pages 1-20. Springer, 2014.

[9] Rosin, P., 1998. Thresholding for change detection, in: 6th Int. Conf. Comput. Vis., Bombay, India. pp. 274-279.

[10] W. T. Freeman, E. C. Pasztor, and O. T. Carmichael. Learning low-level vision. International journal of computer vision, 40(1):25-47, 2000

[11] B. K. Horn and B. G. Schunck. Determining optical flow. In 1981 Technical Symposium East. International Society for Optics and Photonics, 1, pages 319-331981.

[12] H. Scharr and H. Spies. Accurate optical flow in noisy image sequences using flow adapted anisotropic diffusion. Signal Processing: Image Communication, 20(6):537-553, 2005. wavelet

[13] J.Walker, "Combined image compressor and denoiser based on tree adapted shrinkage”, Optical Engineering, Vol.41, P. 1520,2002

[14] Zhu, X., Milanfar, P., 2009. A no-reference sharpnessmetric sensitive to blur and 
noise, in:1st Int.Workshopquality Multimedia Experience, San Diego,CA. pp. 6469.

[15] Jiang, P., Zhang, J., 2015. No-reference image quality assessment based on local maximum gradient. J. Electron. Info. Tech. 37, 2587-2593.

[16] Donoho, D.L., Johnstone, I.M., 1994. Ideal spatialadaptation by wavelet shrinkage.

Biometrika 81, 425- 455. and

[17] Khalil, H., Rahmat, R., Mahmoud, W., 2008. Estimation of noise in gray-scale colored images using median absolute deviation (MAD), in: 3rd Int. Conf. Geometric Model Imaging, London, UK. pp. 92-97.

[18] Ghazi, M.M., Erdogan, H., 2016. Image noise level estimation based on higherorder statistics. Multimed. Tools Appl. 1-19.

[19] Immerkær, J., 1996. Fast noise variance estimation. Comput. Vis. Image Understand. 64, 300-302.

[20] Liu, A., 2009. A fast method of estimating Gaussian noise, in: 1st Int. Conf. Inf. Sci. Eng., pp. 441-444.

[21] Rank, K., Lendl, M., Unbehauen, R., 1999. Estimation of image noise variance, in: IEEE Vis. Image Signal Process., pp. 80-84.

[22] Russo, F., 2007. Gaussian noise estimation in digital images using nonlinear sharpening and genetic optimization, in: Instrum. Meas. Technol. Conf., pp. 1-5.

[23] Tai, S.C., Yang, S.M., 2008. A fast method for image noise estimation using Laplacian operator and adaptive edge detection, in: 3rd Int. Symp. Commun.

Control Signal Process., pp. 1077-1081

[24] Huang, X., Chen, L., Tian, J., Zhang, X., Fu, X., 2013. Blind noisy image quality assessment using block homogeneity. Comput. Electron. Eng. textured

[25] Liu, X., Tanaka, M., Okutomi, M., 2012. Noise level estimation using weak patches of a single noisy image, in: IEEE int. conf. image process., Orlando, USA. pp. 665-668.

[26] S. Pyatykh, J.H., Zheng, L., 2013. Image noise level estimation by principal component analysis. IEEE Trans. Image Process. 22, 687-699.

[27] Tang, C., Yang, X., Zhai, G., 2014. Robust noise estimation based on noise injection. J. Signal Process. Syst. 74, 69-78.

[28] Tian, J., Chen, L., 2012. Image noise estimation using a variation-adaptive evolutionary approach. IEEE Signal Process. Lett. 9, 395-398.

[29] Daniel Zoran, Yair Weiss, "Scale Invariance and Noise in Natural Images”, IEEE 12th International Conference on Computer Vision, 2009.

[30] Ce Liu, Richard Szeliski Sing Bing Kang, "Automatic estimation and removal of noise from a single image" IEEE transactions on pattern analysis and machine intelligence vol.30, No.2, Feb 2008 
[31] Guangmang Cui, Huajun Feng, Zhihai Xu, Qi Li, YuetingChen,”No-reference image noise estimation based on noise level accumulation”

[32] Rafal V Gonzales, Richard E. Woods, and Steven L. Eddins "Digital image processing using MATLAB”, Low price edition, by Pearson education 